\section{Bowel perforation requiring emergency laparo- tomy and a Hartmann's procedure after a gas explosion induced by argon plasma coagulation}

A 95-year-old woman was scheduled to undergo routine argon plasma coagulation (APC) of a bleeding rectal villous adenoma (Figure 1). Bowel preparation was performed using a Fletcher's phosphate enema (Forest Laboratories UK Ltd., Bexley, Kent, UK) approximately 1 hour before the procedure. APC treatment was performed using the Erbe system at the following settings: argon flow rate 1 liter/minute; power $100 \mathrm{~W}$; Endo Cut on; effect 2; spray A65.

A loud explosion was heard on the commencement of treatment. Endoscopic examination revealed the presence of a large amount of blood in the rectum (Figure 2). Immediately, the patient complained of severe abdominal pain; she developed abdominal distension and profuse bleeding per rectum and showed signs of marked hypovolemic shock. This was corrected with infusion of fluids and transfusion of 2 units of packed red cells. A plain radiograph demonstrated generalised pneumoperitoneum.

Preoperative rigid proctosigmoidoscopy showed a large amount of clot in the rectum but no active bleeding. The rectum was unable to retain gas though no perforation could be seen. At laparotomy, there was free intraperitoneal gas but no contamination of the peritoneal cavity. Two full-thickness perforations, with surrounding necrotic bowel wall, were observed in the lower sigmoid colon. This colonic segment was resected with the creation of a proximal end colostomy (Hartmann's procedure). Examination of

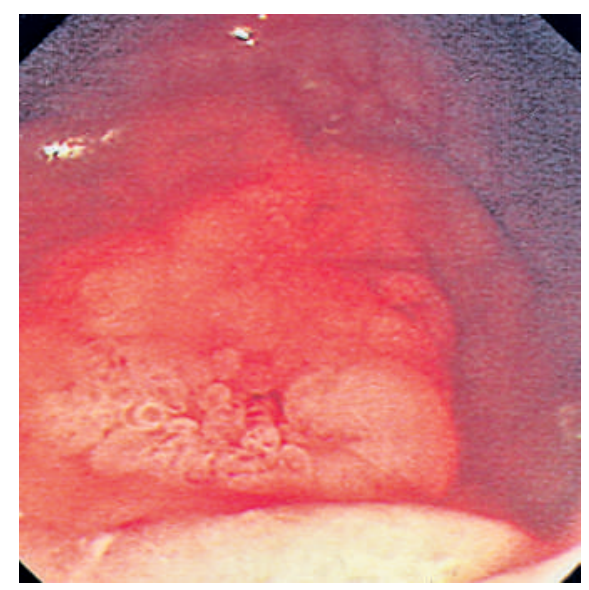

Figure 1 The rectal villous adenoma, $15 \mathrm{~cm}$ from the anal verge, before commencement of treatment.

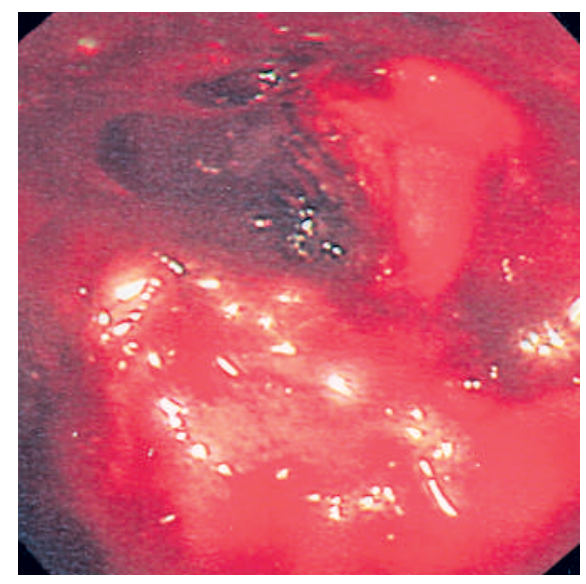

Figure 2 Blood in sigmoid colon after the gas explosion that was induced by argon plasma coagulation.

the surgical specimen revealed multiple areas of necrosis and coagulation, including the two perforation sites.
Postoperatively, her clinical recovery was slow and was complicated by a parastomal hernia that caused small-bowel obstruction but which resolved spontaneously. The patient was discharged 23 days after surgery. Three months later, she was doing well and managing her stoma. It was decided that her stoma would not be reversed because of the location of the resection.

Endoscopy_UCTN_Code_CPL_1AJ_2AZ Endoscopy_UCTN_Code_CPL_1AJ_2AF

\section{A. P. Townshend ${ }^{1}$, W. P. Goddard ${ }^{2}$,}

J. A. Cid ${ }^{1}$

${ }^{1}$ Department of General Surgery, Nottingham City Hospital, Nottingham, United Kingdom

2 Department of Gastroenterology, Nottingham City Hospital, Nottingham, United Kingdom.

\section{Corresponding author}

\section{J. A. Cid, FRCS \\ Nottingham City Hospital \\ Hucknall Road \\ Nottingham, NG5 1PB \\ United Kingdom \\ Email: drjacid@yahoo.es}

